\title{
SIEBENBÜRGISCH-SÄCHSISCHES WÖRTERBUCH.
}

MIT BENÜTZUNG DER SAMMLUNGEN JOHANN WOLFFS

HERAUSGEQEBEN VOM

AUSSCHUSS DES VEREINS FÜR SIEBENBÜRGISCHE LANDESKUNDE.

ERSTER BAND: VIERTE LIEFERUNG

BEARBEITET VON

ADOLF SCHULLERUS

(Batschebierch-Beute).

STRASSBURG

VERLAG VON KARL J. TRÜBNER.

1913.

DRUCK VON W. KRAFFT, HERMANNSTADT. 
Um ein rascheres Erscheinen der einzelnen Lieferungen zu ermöglichen, ist neben dem ersten auch der zweite Band des Siebenbürgisch-sächsischen Wörterbuchs im Druck, so dass nunmehr parallel mit den weiteren Lieferungen des ersten Bandes auch die Lieferungen des zweiten Bandes ausgegeben werden können.

\section{Lesezeichen.}

$\dagger$ nicht mehr gebraucht.

* vor einem Wort bedeutet eine nur erschlossene, nicht bezeugte Form.

, Hauptakzent, 、 Nebenakzent.

> geworden $\mathrm{zu}$, 〈 entstanden aus.

[ ] sprachliche Bemerkungen.

( ) inhaltliche Erläuterungen.

' neuhochdeutsche Umschreibung.

- Zitate aus gedruckten Quellen.

Kursiv mundartliche Redewendungen (ầlt).

Kursivschrift bei Ortsnamen bezeichnet den Ortsnamen als nösnisch (Bistritx).

\section{Fünfter Nachtrag häufiger gebrauchter Abkürzungen.}

B. Capestus Bernhard Capesius, Die Vertreter des alten $\hat{\imath}, \hat{u}, \hat{u}$ im Siebenb.-Sächs. Dissert. Berlin 1912 (Sonderabdruck aus dem Archiv des Vereins für siebenb. Landesk., 38. Bd.).

Engelmans, Viandener Ma. Der Vokalismus der Viandener Mundart von René Engelmann. Diekirch 1910.

Frontus, Flora Flora von Schässburg. Ein Beitrag zur Flora von Siebenbürgen, von Friedr. Fronius, Programm des ev. Gymnasiums in Schässburg. Kronstadt 1858.

Gusinde, Sprachinsel Konrad Gusinde, Eine vergessene deutsche Sprachinsel im polnischen Oberschlesien. (Die Mundart von Schönwald bei Gleiwitz.) Breslau 1911.

KrRCHноF, Volkskunde Beiträge zur Siedlungs- und Volkskunde der Siebenbürger Sachsen. Sonderabdruck aus den Forschungen zur deutschen Landes- und Volkskunde, herausg. von A. Kirchhoff. 9. Bd.

MÜLlRR, Gottesdienst Gottesdienst in einer ev. sächs. Kirche in Siebenbürgen im Jahre 1555. Hermannstadt 1884. (Sonderabdruck aus der Zeitschr. für prakt. Theologie 1884.)

MüNcH Grammatik der ripuarisch-fränkischen Mundart von Ferdinand Münch. Bonn 1904.

TOCKERT, Lehnw. Romanische Lehnwörter in der luxemb. Mundart. Etymologische und kulturhistorische Beiträge zum luxemb. Wörterbuch von Prof. J. Tockert. Luxemburg 1910.

Woeste Fr. Woeste, Wörterbuch der westfäl. Mundart. Norden u. Leipzig 1882. 